\title{
GLOBAL SOLUTIONS OF A STRONGLY COUPLED REACTION-DIFFUSION SYSTEM WITH DIFFERENT DIFFUSION COEFFICIENTS
}

\author{
L. W. SOMATHILAKE AND J. M. J. J. PEIRIS \\ Received 30 January 2004 and in revised form 30 August 2004
}

We deal with a mathematical model for a four-component chemical reaction-diffusion process. The model is described by a system of strongly coupled reaction-diffusion equations with different diffusion rates. The existence of the global solution of this reactiondiffusion system in unbounded domain is proved by using semigroup theory and estimates on the growth of solutions.

\section{Introduction}

In this paper, we prove the existence of a global solution in an unbounded domain of the reaction-diffusion system

$$
\begin{gathered}
\frac{\partial u_{1}}{\partial t}=a_{1} \Delta u_{1}-f_{1}\left(u_{1}, u_{2}, v_{1}, v_{2}\right), \quad x \in \mathbb{R}^{n}, t>0, \\
\frac{\partial u_{2}}{\partial t}=a_{2} \Delta u_{2}-f_{2}\left(u_{1}, u_{2}, v_{1}, v_{2}\right), \quad x \in \mathbb{R}^{n}, t>0, \\
\frac{\partial v_{1}}{\partial t}=b_{1} \Delta u_{1}+d_{1} \Delta v_{1}+f_{3}\left(u_{1}, u_{2}, v_{1}, v_{2}\right), \quad x \in \mathbb{R}^{n}, t>0, \\
\frac{\partial v_{2}}{\partial t}=b_{2} \Delta u_{2}+d_{2} \Delta v_{2}+f_{4}\left(u_{1}, u_{2}, v_{1}, v_{2}\right), \quad x \in \mathbb{R}^{n}, t>0,
\end{gathered}
$$

with the initial conditions

$$
u_{i}(x, 0)=u_{i}^{0}(x), \quad v_{i}(x, 0)=v_{i}^{0}(x) \quad(i=1,2), x \in \mathbb{R}^{n}
$$

Here $f_{1}\left(u_{1}, u_{2}, v_{1}, v_{2}\right)=m\left[k_{2} u_{1}^{m} v_{2}^{r}-k_{1} u_{2}^{r} v_{1}^{m}\right], f_{2}\left(u_{1}, u_{2}, v_{1}, v_{2}\right)=-r\left[k_{2} u_{1}^{m} v_{2}^{r}-k_{1} u_{2}^{r} v_{1}^{m}\right]$, $k_{1} \geq 0, k_{2} \geq 0, m \geq 0, r \geq 1, f_{3}=\rho f_{1}$, and $f_{4}=\rho f_{2}, \rho>0$.

The constants $a_{i}, b_{i}(i=1,2)$ are such that $a_{i}>0, b_{i} \neq 0(i=1,2)$, and $4 a_{i} d_{i}>b_{i}^{2}(i=$ $1,2)$ which reflects the parabolicity of the system. $\Delta$ is the Laplace operator in $\mathbb{R}^{n}$. Moreover we assume that the functions $u_{i}^{0}(i=1,2)$ and $v_{i}^{0}(i=1,2)$ are uniformly bounded, continuous, and nonnegative. This reaction-diffusion system is a mathematical model for 
a chemical reaction of the form

$$
m A+r \bar{B} \underset{k_{2}}{\stackrel{k_{1}}{\rightleftharpoons}} r B+m \bar{A} .
$$

$u_{1}, u_{2}, v_{1}$, and $v_{2}$ represent the concentrations of $\bar{A}, \bar{B}, A$, and $B$, respectively (see [3]).

We remark that the system

$$
\begin{gathered}
\frac{\partial u}{\partial t}=a \Delta u-u h(v), \quad x \in \Omega, t>0, \\
\frac{\partial v}{\partial t}=b \Delta u+d \Delta v+u h(v), \quad x \in \Omega, t>0,
\end{gathered}
$$

with the initial conditions

$$
u(x, 0)=u_{0}(x), \quad v(x, 0)=v_{0}(x), \quad x \in \Omega
$$

on a bounded domain $\Omega \subset \mathbb{R}^{n}$ with Neumann boundary conditions, $b>0, a \neq d, v_{0} \geq$ $(b /(a-d)) u_{0} \geq 0$, and $h(s)$, a differentiable nonnegative function on $\mathbb{R}$, has been studied by Kirane [4]. The existence of global solutions for system (RDS2) on unbounded domains has been studied by Badraoui [1]. The existence of global solutions in $\mathbb{R}^{n}$ for (RDS2) with $h(s)=v^{m}$ has been studied by Collet and Xin [2].

The quasilinear system of reaction-diffusion equations

$$
\begin{gathered}
\frac{\partial u}{\partial t}=\nabla \cdot(a(u) \nabla u)-u h(u) v, \quad x \in \Omega, t>0, \\
\frac{\partial v}{\partial t}=\nabla \cdot(b(v) \nabla v)+u h(u) v-\lambda v, \quad x \in \Omega, t>0,
\end{gathered}
$$

with the initial conditions

$$
u(x, 0)=u_{0}(x), \quad v(x, 0)=v_{0}(x), \quad x \in \Omega,
$$

and with Neumann or Dirichlet boundary conditions, is studied by Kirane [5] where in particular the existence of a globally bounded solution is shown. Also he has discussed large time behavior of the solution.

Our aim is to investigate the existence of a global solution for system (RDS1)-(IC1) in an unbounded domain.

Throughout this paper the following notations are used.

(1) $\|\cdot\|$ is the supremum norm on $\mathbb{R}^{n}$, that is, $\|u\|=\sup _{x \in \mathbb{R}^{n}}|u(x)|$.

(2) $C_{\mathrm{ub}}\left(\mathbb{R}^{n}\right)$ is the space of uniformly bounded continuous functions on $\mathbb{R}^{n}$ equipped with the supnorm.

(3) For any $f \in C_{\mathrm{ub}}\left(\mathbb{R}^{n}\right), \int f=\int_{\mathbb{R}^{n}} f(x) d x$ if the integral exists.

(4) For $f \in L^{p}\left(\mathbb{R}^{n}\right)(p \geq 1),\|f\|_{p}=\left(\int|f|^{p}\right)^{1 / p}$. 


\section{Existence of a local solution}

We convert system (RDS1)-(IC1) to an abstract first-order system in the Banach space $X=\left(C_{\mathrm{ub}}\left(\mathbb{R}^{n}\right)\right)^{4}$ of the form

$$
\begin{gathered}
\frac{d}{d t}(u(t))=A u(t)+F(u(t)), \quad t>0, \\
u(0)=u_{0} \in X,
\end{gathered}
$$

where $u(t)=\left(u_{1}(t), u_{2}(t), v_{1}(t), v_{2}(t)\right)^{T}$. The operator $A$ is defined as

$$
A=\left(\begin{array}{cccc}
a_{1} \Delta & 0 & 0 & 0 \\
0 & a_{2} \Delta & 0 & 0 \\
b_{1} \Delta & 0 & d_{1} \Delta & 0 \\
0 & b_{2} \Delta & 0 & d_{2} \Delta
\end{array}\right)
$$

with domain $D(A)=\left\{u=\left(u_{1}, u_{2}, v_{1}, v_{2}\right)^{T} \in X,\left(\Delta u_{1}, \Delta u_{2}, \Delta v_{1}, \Delta v_{2}\right)^{T} \in X\right\}$.

Moreover the function $F$ is defined as

$$
F(u(t))=\left(-f_{1}(u(t)),-f_{2}(u(t)), f_{3}(u(t)), f_{4}(u(t))\right)^{T},
$$

where

$$
f_{i}(u(t))=f_{i}\left(u_{1}(t), u_{2}(t), v_{1}(t), v_{2}(t)\right), \quad i=1,2,3,4 .
$$

Note that for $\lambda>0$ the operator $\lambda \Delta$ generates an analytic semigroup $G(t)$ in the space $X$ given by

$$
(G(t) u)(x)=\frac{1}{(4 \pi \lambda t)^{n / 2}} \int_{\mathbb{R}^{n}} \exp \left(-\frac{(x-y)^{2}}{4 \lambda t}\right) u(y) d y, \quad t>0, x \in \mathbb{R}^{n}
$$

Let $S_{1}(t), S_{2}(t), S_{3}(t)$, and $S_{4}(t)$ be the semigroups generated by $a_{1} \Delta, a_{2} \Delta, d_{1} \Delta$, and $d_{2} \Delta$, respectively. Then one can show that $A$ generates an analytic semigroup $S(t)$ given by

$$
S(t)=\left(\begin{array}{cccc}
S_{1}(t) & 0 & 0 & 0 \\
0 & S_{2}(t) & 0 & 0 \\
S_{5}(t) & 0 & S_{3}(t) & 0 \\
0 & S_{6}(t) & 0 & S_{4}(t)
\end{array}\right)
$$


where

$$
\begin{aligned}
& S_{5}(t)=\frac{b_{1}}{a_{1}-d_{1}}\left(S_{1}(t)-S_{3}(t)\right), \\
& S_{6}(t)=\frac{b_{2}}{a_{2}-d_{2}}\left(S_{2}(t)-S_{4}(t)\right) .
\end{aligned}
$$

Assume that $F$ is locally Lipschitz in $u$ in the space $X$. Then there exist classical solutions on maximal existence interval $\left[0, T_{0}\right]$ (see $[6]$ ).

\section{Existence of global solutions}

For proving the existence of a global solution we assume that the solutions are nonnegative.

Theorem 3.1. Consider the reaction-diffusion system (RDS1) with nonnegative initial conditions $\left(u_{1}^{0}(x), u_{2}^{0}(x), v_{1}^{0}(x), v_{2}^{0}(x)\right) \in\left(C_{\mathrm{ub}}\left(\mathbb{R}^{n}\right)\right)^{4}, a_{i}>0, d_{i}>a_{i}$, and $b_{i}<0$. Then there exist global in-time classical solutions such that

$$
\left(u_{1}, u_{2}, v_{1}, v_{2}\right) \in\left(C\left([0, \infty) ; C_{\mathrm{ub}}\left(\mathbb{R}^{n}\right)\right) \cap C^{1}\left((0, \infty) ; C_{\mathrm{ub}}\left(\mathbb{R}^{n}\right)\right)\right)^{4}
$$

Lemma 3.2. Let $\left(u_{1}, u_{2}, v_{1}, v_{2}\right)$ be a classical solution of (RDS1). Define the functionals

$$
F^{i}\left(u_{i}, v_{i}\right)=\left(\alpha_{i}+u_{i}+u_{i}^{2}\right) e^{\epsilon_{i} v_{i}} \quad(i=1,2) \text { with } \epsilon_{i}>0, \alpha_{i}>0
$$

Then for any smooth nonnegative function $\psi=\psi(x, t)\left(x \in \mathbb{R}^{n}\right)$ with exponential spatial decay at infinity,

$$
\begin{aligned}
& \frac{d}{d t} \int \psi F^{i}= \int\left(\psi_{t}+d_{i} \Delta \psi\right) F^{i}+\int\left(\left(d_{i}-a_{i}\right) F_{1}^{i}-b_{i} F_{2}^{i}\right) \nabla \psi \nabla u_{i} \\
&-\int \psi\left[\left(a_{i} F_{11}^{i}+b_{i} F_{12}^{i}\right)\left|\nabla u_{i}\right|^{2}+\left(\left(a_{i}+d_{i}\right) F_{12}^{i}+b_{i} F_{22}^{i}\right) \nabla u_{i} \nabla v_{i}\right. \\
&\left.+d_{i} F_{22}^{i}\left(\nabla v_{i}\right)^{2}\right]+\int \psi\left(\rho F_{2}^{i} f_{i}-F_{1}^{i} f_{i}\right), \quad i=1,2
\end{aligned}
$$

where

$$
\begin{gathered}
F_{1}^{i}=\frac{\partial F^{i}}{\partial u_{i}}, \quad F_{2}^{i}=\frac{\partial F^{i}}{\partial v_{i}}, \quad F_{11}^{i}=\frac{\partial^{2} F^{i}}{\partial u_{i}^{2}}, \quad F_{22}^{i}=\frac{\partial^{2} F^{i}}{\partial v_{i}^{2}}, \\
F_{12}^{i}=\frac{\partial^{2} F^{i}}{\partial u_{i} \partial v_{i}}, \quad i=1,2 .
\end{gathered}
$$


Proof. For $i=1,2$, we have

$$
\begin{aligned}
\frac{d}{d t} \int \psi F^{i}= & \int \psi_{t} F^{i}+\int \psi\left(\frac{\partial F^{i}}{\partial u_{i}} \frac{\partial u_{i}}{\partial t}+\frac{\partial F^{i}}{\partial v_{i}} \frac{\partial v_{i}}{\partial t}\right) \\
= & \int \psi_{t} F^{i}+\int \psi\left(F_{1}^{i} \frac{\partial u_{i}}{\partial t}+F_{2}^{i} \frac{\partial v_{i}}{\partial t}\right) \\
= & \int \psi_{t} F^{i}+\int \psi\left(F_{1}^{i}\left(a_{i} \Delta u_{i}-f_{i}\right)+F_{2}^{i}\left(b_{i} \Delta u_{i}+d_{i} \Delta v_{i}+\rho f_{i}\right)\right) \\
= & \int \psi_{t} F^{i}+a_{i} \int \psi F_{1}^{i} \Delta u_{i}+b_{i} \int \psi F_{2}^{i} \Delta u_{i}+d_{i} \int \psi F_{2}^{i} \Delta v_{i} \\
& -\int \psi F_{1}^{i} f_{i}+\rho \int \psi F_{2}^{i} f_{i} .
\end{aligned}
$$

However,

$$
\begin{aligned}
\int \psi F_{1}^{i} \Delta u_{i} & =\int F_{1}^{i} \psi \Delta u_{i} \\
& =-\int \nabla\left(F^{i} \psi\right) \nabla u_{i} \\
& =-\int\left(F_{1}^{i} \nabla \psi+\psi \nabla F_{1}^{i}\right) \nabla u_{i} \\
& =-\int F_{1}^{i} \nabla \psi \nabla u_{i}-\int \psi \nabla F_{1}^{i} \nabla u_{i} \\
& =-\int F_{1}^{i} \nabla \psi \nabla u_{i}-\int \psi\left(F_{11}^{i} \nabla u_{i}+F_{12}^{i} \nabla v_{i}\right) \nabla u_{i},
\end{aligned}
$$

that is,

$$
\int \psi F_{1}^{i} \Delta u_{i}=-\int F_{1}^{i} \nabla \psi \nabla u_{i}-\int \psi F_{11}^{i}\left|\nabla u_{i}\right|^{2}-\int \psi F_{12}^{i} \nabla u_{i} \nabla v_{i} .
$$

Similarly

$$
\begin{aligned}
& \int \psi F_{2}^{i} \Delta u_{i}=-\int F_{2}^{i} \nabla \psi \nabla v_{i}-\int \psi F_{12}^{i}\left|\nabla u_{i}\right|^{2}-\int \psi F_{22}^{i} \nabla u_{i} \nabla v_{i}, \\
& \int \psi F_{2}^{i} \Delta v_{i}=-\int F_{2}^{i} \nabla \psi \nabla v_{i}-\int \psi F_{22}^{i}\left|\nabla v_{i}\right|^{2}-\int \psi F_{12}^{i} \nabla u_{i} \nabla v_{i} .
\end{aligned}
$$

Also

$$
\int F^{i} \Delta \psi=-\int \nabla F^{i} \nabla \psi=-\int F_{1}^{i} \nabla u_{i} \nabla \psi-\int F_{2}^{i} \nabla v_{i} \nabla \psi
$$


Using (3.5)-(3.9) we get

$$
\begin{aligned}
\frac{d}{d t} \int \psi F^{i}= & \int\left(\psi_{t}+d_{i} \Delta \psi\right) F^{i}-a_{i} \int F_{1}^{i} \nabla \psi \nabla u_{i}-a_{i} \int \psi F_{11}^{i}\left|\nabla u_{i}\right|^{2} \\
& -a_{i} \int \psi F_{12}^{i} \nabla u_{i} \nabla v_{i}-b_{i} \int F_{2}^{i} \nabla \psi \nabla u_{i}-b_{i} \int \psi F_{12}^{i}\left|\nabla u_{i}\right|^{2} \\
& -b_{i} \int \psi F_{22}^{i} \nabla u_{i} \nabla v_{i}+d_{i} \int F_{1}^{i} \nabla u_{i} \nabla \psi-d \int \psi F_{22}^{i}\left|\nabla v_{i}\right|^{2} \\
& -d_{i} \int \psi F_{12}^{i} \nabla v_{i} \cdot \nabla v_{i}-\int \psi F_{1}^{i} f_{i}+\rho \int \psi F_{2}^{i} f_{i} \\
= & \int\left(\psi_{t}+d_{i} \Delta \psi\right) F^{i}+\int\left(d_{i} F_{1}^{i}-a_{i} F_{1}^{i}-b_{i} F_{2}^{i}\right) \nabla \psi \nabla u_{i} \\
& -\int\left(a_{i} F_{11}^{i}+b_{i} F_{12}^{i}\right)\left|\nabla u_{i}\right|^{2}-\int \psi\left(a_{i} F_{12}^{i}+b F_{22}^{i}+d_{i} F_{12}^{i}\right) \nabla u_{i} \cdot \nabla v_{i} \\
& -d_{i} \int \psi F_{22}^{i}\left|\nabla v_{i}\right|^{2}-\int \psi F_{1}^{i} f_{i}+\rho \int \psi F_{2}^{i} f_{i} \\
= & \int\left(\psi_{t}+d_{i} \nabla \psi\right) F^{i}+\int\left(\left(d_{i}-a_{i}\right) F_{1}^{i}-b_{i} F_{2}^{i}\right) \nabla \psi \nabla u_{i} \\
& -\int \psi\left[\left(a_{i} F_{11}^{i}+b_{i} F_{12}^{i}\right)\left|\nabla u_{i}\right|^{2}+\left(\left(a_{i}+d_{i}\right) F_{12}^{i}+b_{i} F_{22}^{i}\right) \nabla u_{i} \nabla v_{i}\right. \\
& \left.+d_{i} F_{22}^{i}\left|\nabla v_{i}\right|^{2}\right]+\int \psi\left(\rho F_{2}^{i} f_{i}-F_{1}^{i} f_{i}\right) .
\end{aligned}
$$

Lemma 3.3. There exist four positive constants $\alpha_{i}=\alpha_{i}\left(a_{i}, b, d_{i},\left\|u_{i}^{0}\right\|\right)(i=1,2)$ and $\epsilon_{i}=$ $\epsilon_{i}\left(a_{i}, b_{i}, d_{i},\left\|u_{i}^{0}\right\|\right)(i=1,2)$ such that

$$
\begin{aligned}
\frac{d}{d t} \int \psi F^{i} \leq & \int\left(\psi_{t}+d_{i} \nabla \psi\right) F^{i}+\int\left(\left(d_{i}-a_{i}\right) F_{1}^{i}-b_{i} F_{2}^{i}\right) \nabla \psi \nabla u_{i} \\
& -\frac{1}{2} \int \psi\left[\frac{a_{i}}{2} F_{11}^{i}\left|\nabla u_{i}\right|^{2}+d_{i} F_{22}^{i}\left|\nabla v_{i}\right|^{2}\right]-\frac{1}{2} \int \psi F_{1}^{i} f_{i}, \quad i=1,2 .
\end{aligned}
$$

Proof. For any $\left(u_{i}, v_{i}\right) \in\left[0,\left\|u_{i}^{0}\right\|\right] \times \mathbb{R}^{+}(i=1,2)$, we choose $\alpha_{i}$ and $\epsilon_{i}$ in Lemma 3.2 such that

$$
\begin{gathered}
\rho F_{2}^{i} \leq \frac{1}{2} F_{1}^{i}, \quad i=1,2, \\
\left(a_{i}+d_{i}\right)^{2}\left(F_{12}^{i}\right)^{2}+b_{i}^{2}\left(F_{22}^{i}\right)^{2}+b_{i}\left(2 a_{i}+d_{i}\right)\left(F_{12}^{i}\right)\left(F_{22}^{i}\right)-a_{i} d_{i} F_{11}^{i} F_{22}^{i} \leq 0, \quad i=1,2, \\
\frac{\left(F_{1}^{i}\right)^{2}}{F_{11}^{i}} \leq F^{i}, \quad i=1,2, \\
F_{12}^{i} \leq \frac{a_{i}}{2\left|b_{i}\right|} F_{11}^{i}, \quad i=1,2 .
\end{gathered}
$$


We verify these conditions as follows

$$
\begin{aligned}
F^{i} & =\left(\alpha_{i}+u_{i}+u_{i}^{2}\right) e^{\epsilon_{i} v_{i}}, \quad i=1,2, \\
F_{1}^{i} & =\left(1+2 u_{i}\right) e^{\epsilon_{i} v_{i}}, \quad i=1,2, \\
F_{11}^{i} & =2 e^{\epsilon_{i} v_{i}}, \quad i=1,2, \\
F_{2}^{i} & =\epsilon_{i}\left(\alpha_{i}+u_{i}+u_{i}^{2}\right) e^{\epsilon_{i} v_{i}}, \quad i=1,2, \\
F_{22}^{i} & =\epsilon_{i}^{2}\left(\alpha_{i}+u_{i}+u_{i}^{2}\right) e^{\epsilon_{i} v_{i}}, \quad i=1,2, \\
F_{12} & =F_{21}^{i}=\epsilon_{i}\left(1+2 u_{i}\right) e^{\epsilon_{i} v_{i}}, \quad i=1,2 .
\end{aligned}
$$

Denote

$$
\begin{aligned}
\epsilon_{i}^{(1)} & =\frac{1}{2 \rho\left(\alpha_{i}+\left\|u_{i}^{0}\right\|+\left\|u_{i}^{0}\right\|^{2}\right)}, \\
\epsilon_{i}^{(2)} & =\frac{1}{b_{i}\left(\alpha_{i}+\left\|u_{i}^{0}\right\|+\left\|u_{i}^{0}\right\|^{2}\right)}, \\
\epsilon_{i}^{(3)} & =\frac{1}{\left|b_{i}\right|\left(1+2\left\|u_{i}^{0}\right\|\right)}, \\
\alpha_{i}^{(1)} & =\frac{\left(a_{i}+d_{i}\right)^{2}\left(1+2\left\|u_{i}^{0}\right\|\right)^{2}+\left(2 a_{i}+d_{i}\right)\left(1+2\left\|u_{i}^{0}\right\|\right)}{2 a_{i} d_{i}}, \\
\alpha_{i}^{(2)} & =\frac{1+2\left\|u_{i}^{0}\right\|^{2}+2\left\|u_{i}^{0}\right\|}{2}=\frac{\left(1+\left\|u_{i}^{0}\right\|\right)^{2}+\left\|u_{i}^{0}\right\|^{2}}{2} .
\end{aligned}
$$

If we choose $\epsilon_{i}(i=1,2)$ such that $\epsilon_{i} \leq \epsilon_{i}^{(1)}(i=1,2)$ then condition (3.12) is satisfied.

Condition (3.13) is satisfied if

$$
\begin{gathered}
\left(a_{i}+d_{i}\right)^{2} \epsilon_{i}^{2}\left(1+2 u_{i}\right)^{2} e^{2 \epsilon_{i} v_{i}}+b_{i}^{2} \epsilon_{i}^{4}\left(\alpha_{i}+u_{i}+u_{i}^{2}\right)^{2} e^{2 \epsilon_{i} v_{i}}+b_{i}\left(2 a_{i}+d_{i}\right) \epsilon_{i}^{3}\left(1+2 u_{i}\right) \\
\times\left(\alpha_{i}+u_{i}+u_{i}^{2}\right)^{2} e^{2 \epsilon_{i} v_{i}}-2 a_{i} d_{i} \epsilon_{i}^{2}\left(\alpha_{i}+u_{i}+u_{i}^{2}\right) e^{2 \epsilon_{i} v_{i}} \leq 0, \quad i=1,2,
\end{gathered}
$$

that is,

$$
\begin{aligned}
& \left(a_{i}+d_{i}\right)^{2}\left(1+2 u_{i}\right)^{2}+b_{i}^{2} \epsilon_{i}^{2}\left(\alpha_{i}+u_{i}+u_{i}^{2}\right)^{2} \\
& \quad+b_{i}\left(2 a_{i}+d_{i}\right)\left(1+2 u_{i}\right)\left(\alpha_{i}+u_{i}+u_{i}^{2}\right) \epsilon_{i}-2 a_{i} d_{i}\left(\alpha_{i}+u_{i}+u_{i}^{2}\right) \leq 0 .
\end{aligned}
$$

If we choose $\epsilon_{i}(i=1,2)$ and $\alpha_{i}(i=1,2)$ such that $\epsilon_{i} \leq \epsilon_{i}^{(2)}(i=1,2)$ and $\alpha_{i} \leq \alpha_{i}^{(1)}(i=$ $1,2)$, then (3.19) is satisfied. In other words, condition (3.13) is satisfied.

Condition (3.14) is satisfied if

$$
\frac{\left(1+2 u_{i}\right)^{2}}{2} \leq\left(\alpha_{i}+u_{i}+u_{i}^{2}\right)
$$

that is,

$$
\alpha_{i}>\frac{1+2 u_{i}^{2}+2 u_{i}}{2} .
$$


If we choose $\alpha_{i}(i=1,2)$ such that $\alpha_{i} \leq \alpha_{i}^{(2)}(i=1,2)$, then (3.21) is satisfied. Hence condition (3.14) is satisfied.

Similarly we can show that condition (3.15) is satisfied if $\epsilon_{i}(i=1,2)$ is chosen such that $\epsilon_{i} \leq \epsilon_{i}^{(3)}$.

Now select

$$
\begin{aligned}
& \alpha_{i} \geq \max \left(\alpha_{i}^{(1)}, \alpha_{i}^{(2)}\right) \quad(i=1,2), \\
& \epsilon_{i} \leq \min \left(\epsilon_{i}^{(1)}, \epsilon_{i}^{(2)}, \epsilon_{i}^{(3)}\right) \quad(i=1,2) .
\end{aligned}
$$

Then conditions (3.12)-(3.15) are satisfied.

Then, from (3.13) and (3.15), we get

$$
\begin{aligned}
\left(a_{i} F_{11}^{i}\right. & \left.+b_{i} F_{12}^{i}\right)\left|\nabla u_{i}\right|^{2}+\left(\left(a_{i}+d_{i}\right) F_{12}^{i}+b_{i} F_{22}^{i}\right) \nabla u_{i} \nabla v_{i}+d_{i} F_{22}^{i}\left|\nabla v_{i}\right|^{2} \\
& \geq \frac{1}{2}\left[\left(a_{i} F_{11}^{i}+b_{i} F_{12}^{i}\right)\left|\nabla u_{i}\right|^{2}+d_{i} F_{22}^{i}\left|\nabla v_{i}\right|^{2}\right] \\
& \geq \frac{1}{2}\left[\frac{a_{i}}{2} F_{11}^{i}\left|\nabla u_{i}\right|^{2}+d_{i} F_{22}^{i}\left|\nabla v_{i}\right|^{2}\right] .
\end{aligned}
$$

From (3.12), we get

$$
\int \psi\left(\rho F_{2}^{i} f_{i}-F_{1}^{i} f_{i}\right) \leq-\frac{1}{2} \int \psi F_{1}^{i} f_{i}, \quad i=1,2
$$

From (3.3), (3.23), (3.24), we get (3.11).

The proof of Lemma 3.3 is completed.

Theorem 3.4. If $\alpha_{i}, \epsilon_{i}(i=1,2)$ satisfy (3.22), then there exist a test function $\psi$ and real positive constants $\beta_{i}(i=1,2)$ and $\sigma_{i}$ such that

$$
\int \psi F_{i} \leq \beta_{i} e^{\sigma_{i} t}, \quad \forall t>0, i=1,2
$$

Proof. We define the test function $\psi(x)$ as

$$
\psi(x)=\frac{1}{\left(1+\left|x-x_{0}\right|^{2}\right)^{n}}, \quad x \in \mathbb{R}^{n},
$$

and $x_{0} \in \mathbb{R}^{n}$ is an arbitrary point.

Then $\psi$ is a smooth function with exponential decay at infinity and satisfies $|\Delta \psi| \leq$ $K_{1} \psi,|\nabla \psi| \leq K_{2} \psi$. Let $K=\max \left(K_{1}, K_{2}\right)$ for some positive constant $K$. 
Then from (3.11), we obtain

$$
\begin{aligned}
\frac{d}{d t} \int \psi F^{i} \leq & K d_{i} \int \psi F^{i}+K\left(\left(d_{i}-a_{i}\right)+\frac{1}{2}\left|b_{i}\right|\right) \int F_{1}^{i} \psi\left|\nabla u_{i}\right| \\
& -\frac{a_{i}}{4} \int \psi F_{11}^{i}|\nabla u|^{2}-\frac{d_{i}}{2} \int \psi F_{22}^{i}\left|\nabla v_{i}\right|^{2}-\frac{1}{2} \int \psi F_{1}^{i} f_{i} \\
\leq & K d_{i} \int \psi F^{i}+\frac{K^{2}}{a_{i}}\left(\left(d_{i}-a_{i}\right)+\frac{1}{2}\left|b_{i}\right|\right)^{2} \int \psi \frac{\left(F_{1}^{i}\right)^{2}}{F_{11}^{i}} \\
\leq & {\left[K d_{i}+\frac{K^{2}}{a_{i}}\left(\left(d_{i}-a_{i}\right)+\frac{1}{2}\left|b_{i}\right|^{2}\right)^{2}\right] \int \psi F^{i}, \quad i=1,2 . }
\end{aligned}
$$

Let

$$
\begin{aligned}
& \sigma_{i}=K d_{i}+\frac{K^{2}}{a_{i}}\left(\left|d_{i}-a_{i}\right|+\frac{1}{2}\left|b_{i}\right|^{2}\right)^{2}, \quad i=1,2, \\
& \beta_{i} \geq\left(\alpha_{i}+\left\|u_{i}^{0}\right\|+\left\|u_{i}^{0}\right\|^{2}\right) e^{\epsilon\left\|v_{i}^{0}\right\|}\|\psi\|_{1}, \quad i=1,2 .
\end{aligned}
$$

Then

$$
\frac{d}{d t} \int \psi F^{i} \leq \sigma_{i} \int \psi F^{i} \quad \text { for } i=1,2
$$

which implies (3.25).

Lemma 3.5. For any unit cube $Q$ and any finite $p \geq 1$,

$$
\int_{Q}\left|v_{i}\right|^{p} d x \leq 2^{n} \frac{\beta_{i}}{\alpha_{i} \epsilon_{i}^{p}} e^{\sigma_{i} t}(p+1)^{p+1} \quad \text { for } i=1,2 .
$$

Proof. Using the results in Theorem 3.4, for any nonnegative integer $p$ we have

$$
\beta_{i} e^{\sigma_{i} t} \geq \int \psi F^{i} \geq \alpha_{i} \int \psi e^{\epsilon_{i} v_{i}} \geq \alpha_{i} \epsilon_{i}^{p} \int_{Q} \psi \frac{v_{i}^{p}}{p !}, \quad t>0, i=1,2
$$

By taking $x_{0}$ at the center of $Q$, we get

$$
\beta_{i} e^{\sigma_{i} t} \geq \frac{\alpha_{i} \epsilon_{i}^{p}}{p !} \int_{Q} \frac{v_{i}^{p}}{2^{n}} \geq \frac{\alpha_{i} \epsilon_{i}^{p}}{2^{n}(p+1)^{p+1}} \int_{Q} v_{i}^{p}, \quad i=1,2 .
$$

This implies (3.30). 
Lemma 3.6. There exist constants $c_{i}=c_{i}\left(n, \lambda,\left\|u_{i}^{0}\right\|,\left\|v_{3-i}^{0}\right\|, t\right), i=1,2$ such that

$$
\begin{aligned}
G(t-s) * u_{i}^{r}(x, s) v_{3-i}^{m}(x, s) & =\frac{1}{(4 \pi \lambda(t-s))^{n / 2}} \int e^{-|x-y|^{2} / 4 \lambda(t-s)} u_{i}^{r}(y, s) v_{3-i}^{m}(y, s) d y \\
& \leq c_{i}\left((t-s)^{n / 2 q}+(t-s)^{-n / 2 p}\right), \quad i=1,2
\end{aligned}
$$

for any $p>\max \{1, n / 2\}, 1 / p+1 / q=1$. Here $G(t)$ is the semigroup generated by the operator $\lambda \Delta,(\lambda>0)$ on the space $C_{\mathrm{ub}}\left(\mathbb{R}^{n}\right)$.

Proof. Let $\left\{Q_{j}\right\}, j=0,1,2, \ldots$, be the tilling of $\mathbb{R}^{n}$ by unit cubes $Q_{j}$ 's such that $x$ is at the center of $Q_{0}$. Then

$$
\int e^{-(x-y)^{2} / 4 \lambda(t-s)} u_{i}^{r}(y, s) v_{3-i}^{m}(y, s) d y=\sum_{Q_{j}} \int_{Q_{j}} e^{-(x-y)^{2} / 4 \lambda(t-s)} u_{i}^{r}(y, s) v_{3-i}^{m}(y, s) d y .
$$

For $y \in Q_{j}$ we have the inequality

$$
e^{-(x-y)^{2} / 8 \lambda(t-s)} \leq \sup _{y \in Q_{j}} e^{-(x-y)^{2} / 8 \lambda(t-s)}=e^{-\operatorname{dist}^{2}\left(x, Q_{j}\right) / 8 \lambda(t-s)} .
$$

Also there exists a positive constant $c(n)$ such that if $y \in Q_{j}, j \neq 0$, we have

$$
c(n) \operatorname{dist}^{2}\left(x, Q_{j}\right) \geq(x-y)^{2} .
$$

Let $I_{1}=\int e^{-(x-y)^{2} / 8 \lambda(t-s)} u_{i}^{r}(y, s) v_{3-i}^{m}(y, s) d y$. Then applying Hölder's inequality with $p \geq$ $n / 2$ and its conjugate $q$, we get

$$
\begin{aligned}
I_{1} & \leq\left(\int_{Q_{j}} e^{-q(x-y)^{2} / 8 \lambda(t-s)}\right)^{1 / q}\left(\int_{Q_{j}} u_{i}^{r p}(y, s) v_{3-i}^{m p}(y, s) d y\right)^{1 / p} \\
& \leq\left(\int_{Q_{j}} e^{-q \operatorname{dist}^{2}\left(x, Q_{j}\right) / 8 \lambda(t-s)}\right)^{1 / q}\left(\int_{Q_{j}} u_{i}^{r p}(y, s) v_{3-i}^{m p}(y, s) d y\right)^{1 / p} \\
& \leq \frac{(8 \pi \lambda)^{n / 2 q}(t-s)^{n / 2 q}}{q}\left(\int_{Q_{j}} u_{i}^{r p}(y, s) v_{3-i}^{m p}(y, s) d y\right)^{1 / p} \\
& \leq \frac{(8 \pi \lambda)^{n / 2 q}(t-s)^{n / 2 q}}{q}\left(\int_{Q_{j}}\left|u_{i}\right|^{r p}\left|v_{3-i}\right|^{m p} d y\right)^{1 / p} \\
& \leq \frac{(8 \pi \lambda)^{n / 2 q}(t-s)^{n / 2 q}}{q}\left\|u_{i}^{0}\right\|^{r}\left(\int_{Q_{j}}\left|v_{3-i}\right|^{m p} d y\right)^{1 / p} \\
& \leq \frac{(8 \pi \lambda)^{n / 2 q}(t-s)^{n / 2 q}}{q}\left\|u_{i}^{0}\right\|^{r}\left(2^{m p} \frac{\beta_{i}}{\alpha_{i} \epsilon_{i}^{m p}} e^{\sigma t}(m p+1)^{m p+1}\right)^{1 / p} \\
& =\frac{(8 \pi \lambda)^{n / 2 q}(t-s)^{n / 2 q}}{q}\left\|u_{i}^{0}\right\|^{r} 2^{m}\left(\frac{\beta_{i}}{\alpha_{i}}\right)^{1 / p} e^{\sigma t / p} \epsilon_{i}^{-m}(m p+1)^{m+1 / p} .
\end{aligned}
$$


That is

$$
\int e^{-(x-y)^{2} / 8 \lambda(t-s)} u_{i}^{r}(y, s) v_{3-i}^{m}(y, s) d y \leq c_{i}^{1}\left(n, \lambda,\left\|u_{i}^{0}\right\|,\left\|v_{3-i}^{0}\right\|, t\right)(t-s)^{n / 2 q}, \quad i=1,2
$$

where

$$
c_{i}^{1}\left(n, \lambda,\left\|u_{i}^{0}\right\|,\left\|v_{3-i}^{0}\right\|, t\right)=\frac{(8 \pi \lambda)^{n / 2 q}}{q}\left\|u_{i}^{0}\right\|^{r} 2^{m}\left(\frac{\beta_{i}}{\alpha_{i}}\right)^{1 / p} e^{\sigma t / p} \epsilon_{i}^{-m}(m p+1)^{m+1 / p}, \quad i=1,2 .
$$

Now we have

$$
\begin{aligned}
I_{2} & =G(t-s) * u_{i}^{r}(x, s) v_{3-i}^{m}(x, s) \\
& \leq \frac{1}{(4 \pi \lambda(t-s))^{n / 2}} \sum_{Q_{j}} e^{-\operatorname{dist}^{2}\left(x, Q_{j}\right) / 8 \lambda(t-s)} \int e^{-|x-y|^{2} / 8 \lambda(t-s)} u_{i}^{r}(y, s) v_{3-i}^{m}(y, s) d y \\
& \leq \frac{1}{(4 \pi \lambda(t-s))^{n / 2}} c_{i}^{1}\left(n, \lambda,\left\|u_{i}^{0}\right\|,\left\|v_{3-i}^{0}\right\|, t\right)(t-s)^{n / 2 q} \sum_{Q_{j}} e^{-\operatorname{dist}^{2}\left(x, Q_{j}\right) / 8 \lambda(t-s)} \\
& \leq c_{i}\left(n, \lambda,\left\|u_{i}^{0}\right\|,\left\|v_{3-i}^{0}\right\|, t\right)(t-s)^{-n / 2 p}\left(\int e^{-(x-y)^{2} / 8 \lambda c(n)(t-s)} d y+1\right) \\
& \leq c_{i}\left(n, \lambda,\left\|u_{i}^{0}\right\|,\left\|v_{3-i}^{0}\right\|, t\right)(t-s)^{-n / 2 p}\left(8 \pi \lambda c(n)(t-s)^{n / 2}+1\right) \\
& \leq c_{i}\left(n, \lambda,\left\|u_{i}^{0}\right\|,\left\|v_{3-i}^{0}\right\|, t\right)\left((t-s)^{n / 2 q}+(t-s)^{-n / 2 p}\right),
\end{aligned}
$$

where $c_{i}\left(n, \lambda,\left\|u_{i}^{0}\right\|,\left\|v_{3-i}^{0}\right\|, t\right)=c_{i}^{1}\left(n, \lambda,\left\|u_{i}^{0}\right\|,\left\|v_{3-i}^{0}\right\|, t\right)\left(1 /(4 \pi \lambda)^{n / 2}\right)$.

The integral formula for $u(t)$ is

$$
u(t)=S(t) u_{0}+\int_{0}^{t} S(t-s) F(u(s)) d s
$$

From (3.41) we can deduce that

$$
\begin{aligned}
& u_{i}(x, t)=S_{i}(t) u_{i}^{0}-\int_{0}^{t} S_{i}(t-s) f_{i}(u(s)) d s, \quad i=1,2, \\
& v_{1}(x, t)=S_{5}(t) u_{1}^{0}+S_{3}(t) v_{1}^{0}-\int_{0}^{t} S_{5}(t-s) f_{1}(u(s)) d s+\rho \int_{0}^{t} S_{3}(t-s) f_{1}(u(s)) d s, \\
& v_{2}(x, t)=S_{6}(t) u_{2}^{0}+S_{4}(t) v_{2}^{0}-\int_{0}^{t} S_{6}(t-s) f_{1}(u(s)) d s+\rho \int_{0}^{t} S_{4}(t-s) f_{1}(u(s)) d s .
\end{aligned}
$$


From (2.7) and (3.43), we get

$$
\begin{aligned}
v_{1}(x, t)= & \frac{b_{1}}{a_{1}-d_{1}}\left(S_{1}(t)-S_{3}(t)\right) u_{1}^{0}+S_{3}(t) v_{1}^{0} \\
& -\int_{0}^{t} \frac{b_{1}}{a_{1}-d_{1}}\left(S_{1}(t-s)-S_{3}(t-s)\right) f_{1}(u(s)) d s \\
& +\rho \int_{0}^{t} S_{3}(t-s) f_{1}(u(s)) d s \\
= & \frac{b_{1}}{a_{1}-d_{1}} S_{1}(t) u_{1}^{0}+S_{3}(t)\left(v_{1}^{0}-\frac{b_{1}}{a_{1}-d_{1}} u_{1}^{0}\right)-\frac{b_{1}}{a_{1}-d_{1}} \int_{0}^{t} S_{1}(t-s) f_{1}(u(s)) d s \\
& +\left(\rho+\frac{b_{1}}{a_{1}-d_{1}}\right) \int_{0}^{t} S_{3}(t-s) f_{1}(u(s)) d s .
\end{aligned}
$$

Hence

$$
\begin{aligned}
v_{1}(x, t)= & \frac{b_{1}}{a_{1}-d_{1}} S_{1}(t) u_{1}^{0}+S_{3}(t)\left(v_{1}^{0}-\frac{b_{1}}{a_{1}-d_{1}} u_{1}^{0}\right) \\
& +\frac{b_{1}}{a_{1}-d_{1}} \int_{0}^{t} S_{1}(t-s)\left(m k_{1} u_{2}^{r}(s) v_{1}^{m}(s)\right) d s \\
& -\frac{b_{1}}{a_{1}-d_{1}} \int_{0}^{t} S_{1}(t-s)\left(m k_{2} u_{1}^{m}(s) v_{2}^{r}(s)\right) d s \\
& -\left(\rho+\frac{b_{1}}{a_{1}-d_{1}}\right) \int_{0}^{t} S_{3}(t-s)\left(m k_{1} u_{2}^{r}(s) v_{1}^{m}(s)\right) d s \\
& +\left(\rho+\frac{b_{1}}{a_{1}-d_{1}}\right) \int_{0}^{t} S_{3}(t-s)\left(m k_{2} u_{1}^{m}(s) v_{2}^{r}(s)\right) d s
\end{aligned}
$$

that is,

$$
\begin{aligned}
\left|v_{1}(x, t)\right|= & \frac{b_{1}}{a_{1}-d_{1}}\left|S_{1}(t) u_{1}^{0}\right|+\left|S_{3}(t)\left(v_{1}^{0}-\frac{b_{1}}{a_{1}-d_{1}} u_{1}^{0}\right)\right| \\
& +\frac{b_{1}}{a_{1}-d_{1}}\left|\int_{0}^{t} S_{1}(t-s)\left(m k_{1} u_{2}^{r}(s) v_{1}^{m}(s)\right) d s\right| \\
& +\frac{b_{1}}{a_{1}-d_{1}}\left|\int_{0}^{t} S_{1}(t-s)\left(m k_{2} u_{1}^{m}(s) v_{2}^{r}(s)\right) d s\right| \\
& +\left(\rho+\frac{b_{1}}{a_{1}-d_{1}}\right)\left|\int_{0}^{t} S_{3}(t-s)\left(m k_{1} u_{2}^{r}(s) v_{1}^{m}(s)\right) d s\right| \\
& +\left(\rho+\frac{b_{1}}{a_{1}-d_{1}}\right)\left|\int_{0}^{t} S_{3}(t-s)\left(m k_{2} u_{1}^{m}(s) v_{2}^{r}(s)\right) d s\right|
\end{aligned}
$$


Using relation (3.33) and the property that $S_{1}(t)$ and $S_{3}(t)$ are contraction semigroups, we get

$$
\begin{aligned}
\left\|v_{1}(x, t)\right\| \leq & \frac{b_{1}}{a_{1}-d_{1}}\left\|u_{1}^{0}\right\|+\left\|v_{1}^{0}\right\| \\
& +\frac{m b_{1}}{a_{1}-d_{1}}\left(k_{1} c_{1}\left(n, a_{1},\left\|u_{2}^{0}\right\|,\left\|v_{1}^{0}\right\|, t\right)+k_{2} c_{2}\left(n, a_{1},\left\|u_{1}^{0}\right\|,\left\|v_{2}^{0}\right\|, t\right)\right) \\
& \times\left(\int_{0}^{t}\left((t-s)^{n / 2 q}+(t-s)^{-n / 2 p}\right) d s\right) \\
& +\left(\rho+\frac{m b_{1}}{a_{1}-d_{1}}\right)\left(k_{1} c_{3}\left(n, d_{1},\left\|u_{2}^{0}\right\|,\left\|v_{1}^{0}\right\|, t\right)+k_{2} c_{4}\left(n, d_{1},\left\|u_{1}^{0}\right\|,\left\|v_{1}^{0}\right\|, t\right)\right) \\
& \times\left(\int_{0}^{t}\left((t-s)^{n / 2 q}+(t-s)^{-n / 2 p}\right) d s\right), \\
\left\|v_{1}(x, t)\right\| \leq & \frac{b_{1}}{a_{1}-d_{1}}\left\|u_{1}^{0}\right\|+\left\|v_{1}^{0}\right\|+\frac{m b_{1}}{a_{1}-d_{1}}\left(k_{1} c_{1}+k_{2} c_{2}\right)\left|\int_{0}^{t}\left((t-s)^{n / 2 q}+(t-s)^{-n / 2 p}\right) d s\right| \\
& +m\left(\rho+\frac{b_{1}}{a_{1}-d_{1}}\right)\left(k_{1} c_{3}+k_{2} c_{4}\right)\left|\int_{0}^{t}\left((t-s)^{n / 2 q}+(t-s)^{-n / 2 p}\right) d s\right| .
\end{aligned}
$$

If $p>n / 2$, we get

$$
\begin{aligned}
\left\|v_{1}(x, t)\right\| \leq & \frac{b_{1}}{a_{1}-d_{1}}\left\|u_{1}^{0}\right\|+\left\|v_{1}^{0}\right\|+\frac{m b_{1}}{a_{1}-d_{1}}\left(k_{1} c_{1}+k_{2} c_{2}\right)\left(\frac{2 q t^{n / 2 q+1}}{n+2 q}+\frac{2 p t^{1-n / 2 p}}{2 p-n}\right) \\
& +m\left(\rho+\frac{b_{1}}{a_{1}-d_{1}}\right)\left(k_{1} c_{3}+k_{2} c_{4}\right)\left(\frac{2 q t^{n / 2 q+1}}{2 q+n}+\frac{2 p t^{1-n / 2 p}}{2 p-n}\right), \quad t>0 .
\end{aligned}
$$

Similarly

$$
\begin{aligned}
\left\|v_{2}(x, t)\right\| \leq & \frac{b_{2}}{a_{2}-d_{2}}\left\|u_{1}^{0}\right\|+\left\|v_{1}^{0}\right\|+\frac{r b_{2}}{a_{2}-d_{2}}\left(k_{1} c_{5}+k_{2} c_{6}\right)\left(\frac{2 q t^{n / 2 q+1}}{n+2 q}+\frac{2 p t^{1-n / 2 p}}{2 p-n}\right) \\
& +r\left(\rho+\frac{b_{2}}{a_{2}-d_{2}}\right)\left(k_{1} c_{7}+k_{2} c_{8}\right)\left(\frac{2 q t^{n / 2 q+1}}{2 q+n}+\frac{2 p t^{1-n / 2 p}}{2 p-n}\right), \quad t>0 .
\end{aligned}
$$

Similarly one can show that

$$
\begin{array}{ll}
\left\|u_{1}(x, t)\right\| \leq\left\|u_{1}^{0}\right\|+m\left(k_{1} c_{9}+k_{2} c_{10}\right)\left(\frac{2 q t^{n / 2 q+1}}{2 q+n}+\frac{2 p t^{1-n / 2 p}}{2 p-n}\right), \quad t>0, \\
\left\|u_{2}(x, t)\right\| \leq\left\|u_{2}^{0}\right\|+m\left(k_{1} c_{11}+k_{2} c_{12}\right)\left(\frac{2 q t^{n / 2 q+1}}{2 q+n}+\frac{2 p t^{1-n / 2 p}}{2 p-n}\right), \quad t>0 .
\end{array}
$$

Here $c_{1}-c_{12}$ are constants.

From estimates (3.49)-(3.52) and the standard parabolic regularity theory we get the existence of global classical solution $\left(u_{1}, u_{2}, v_{1}, v_{2}\right) \in\left(C\left([0, \infty), C_{\mathrm{ub}}\right) \cap C^{1}\left((0, \infty), C_{\mathrm{ub}}\right)\right)^{4}$. 


\section{Acknowledgments}

Dr. J. M. J. J. Peiris, one of the authors of this paper, whose contribution to the present work was immense, passed away under tragic circumstances on 16 January 2004. Hence this paper is dedicated to him.

\section{References}

[1] S. Badraoui, Existence of global solutions for systems of reaction-diffusion equations on unbounded domains, Electron. J. Differential Equations 2002 (2002), no. 74, 1-10.

[2] P. Collet and J. Xin, Global existence and large time asymptotic bounds of $L^{\infty}$ solutions of thermal diffusive combustion systems on $\mathbb{R}^{n}$, Ann. Scuola Norm. Sup. Pisa Cl. Sci. (4) 23 (1996), no. 4, 625-642.

[3] C. Jiang and C. Xie, Global bounded solutions of a strongly coupled reaction-diffusion system, Nonlinear Stud. 7 (2000), no. 2, 227-240.

[4] M. Kirane, Global bounds and asymptotics for a system of reaction-diffusion equations, J. Math. Anal. Appl. 138 (1989), no. 2, 328-342.

[5] Global pointwise a priori bounds and large time behaviour for a nonlinear system describing the spread of infectious disease, Appl. Math. (Warsaw) 22 (1993), no. 1, 1-9.

[6] A. Pazy, Semigroups of Linear Operators and Applications to Partial Differential Equations, Springer-Verlag, New York, 1993.

L. W. Somathilake: Department of Mathematics, University of Ruhuna, Matara, Sri Lanka

E-mail address: sthilake@maths.ruh.ac.lk

J. M. J. J. Peiris: Department of Mathematics, University of Ruhuna, Matara, Sri Lanka 


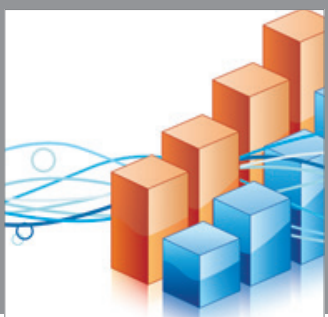

Advances in

Operations Research

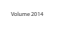

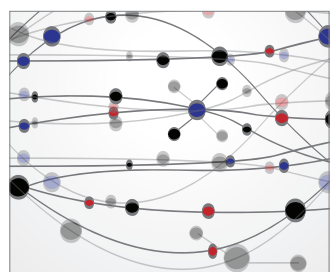

\section{The Scientific} World Journal
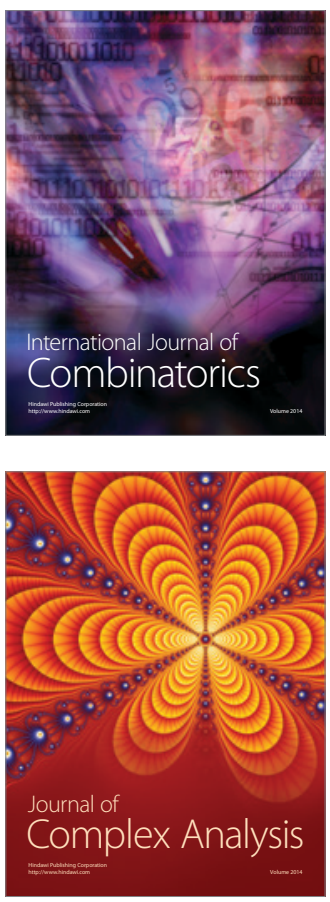

International Journal of

Mathematics and

Mathematical

Sciences
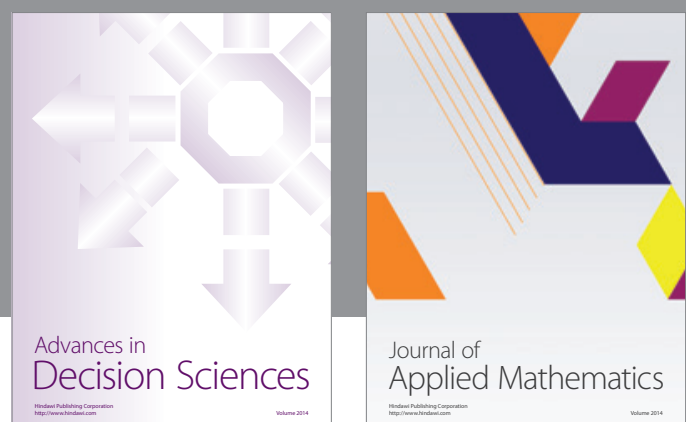

Journal of

Applied Mathematics
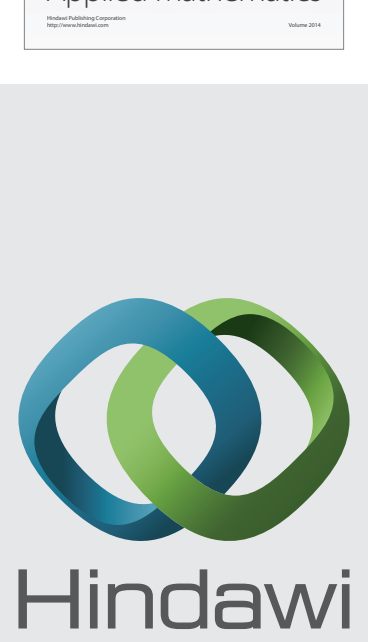

Submit your manuscripts at http://www.hindawi.com
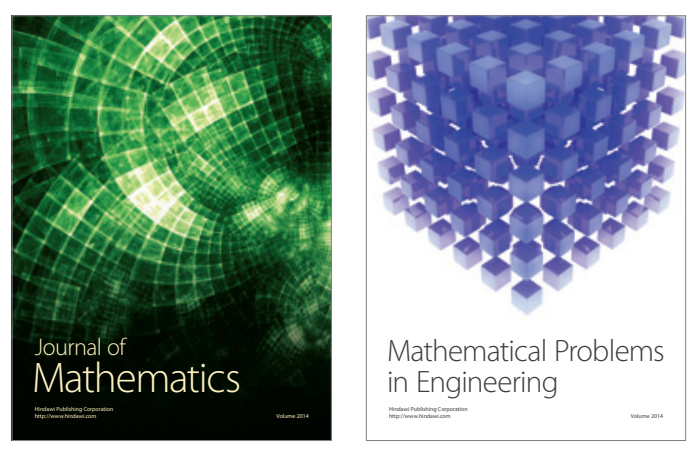

Mathematical Problems in Engineering
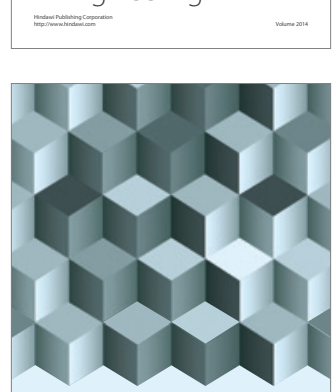

Journal of

Function Spaces
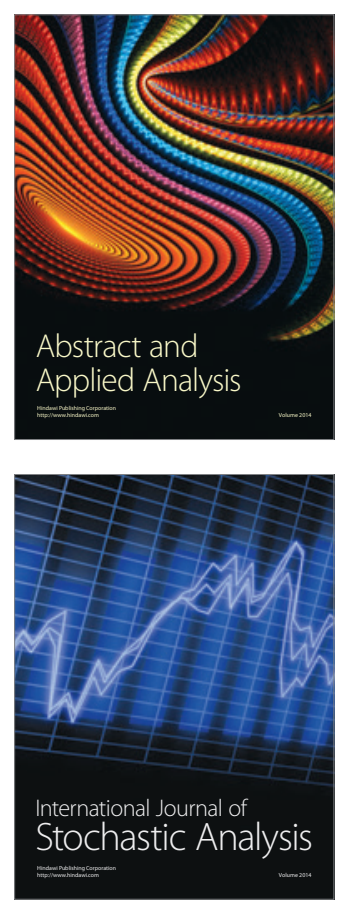

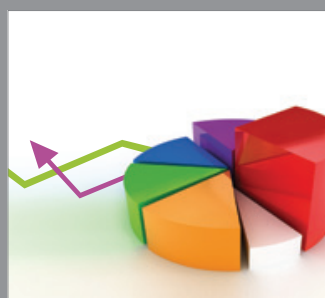

ournal of

Probability and Statistics

Promensencen
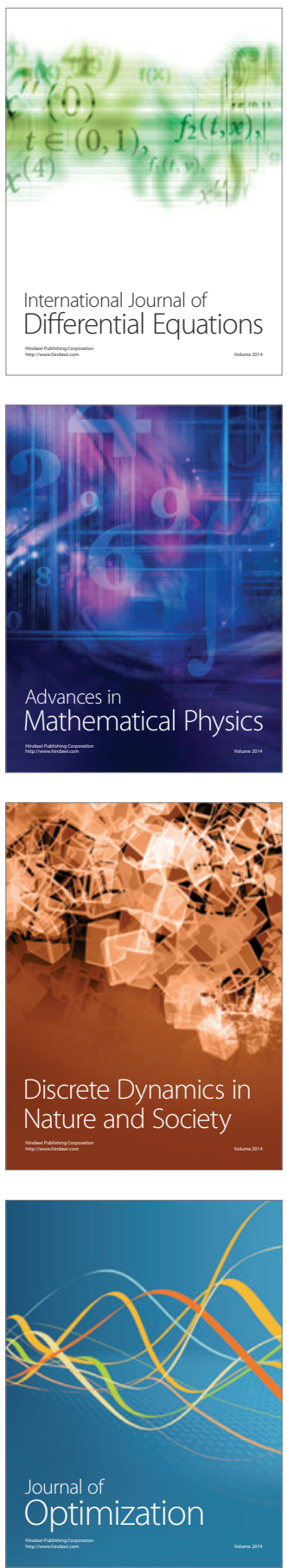